\title{
Construction of recombinant ORFV-TRAP using CRISPR/Cas9 technology
}

\author{
Yongzhong Yu ${ }^{1}$, Fan Zhang ${ }^{1}$, Xuyang Duan ${ }^{1}$, Chaoqun Yang ${ }^{1}$, Zhengxing Lian ${ }^{1}$, and \\ Yudong $\mathrm{Cui}^{1}$
}

${ }^{1}$ Affiliation not available

January 25, 2022

\begin{abstract}
Orf is an acute and highly contracted human and animal infection caused by orf virus (ORFV), which mainly affects sheep and goats, especially young animals, who have close contact with sick animals. Clinically, in secondary infection cases of orf, opportunistic or conditional pathogens such as Staphylococcus aureus (S. aureus) are often detected. The S. aureus TRAP gene product is reportedly protective against bacterial infection. For joint control of ORFV and S. aureus, the research direction of delivering a TRAP gene vaccine against S. aureus infection using the ORFV live vector is proposed. Here, we used CRISPR/Cas9 technology to edit vascular endothelial growth factor E of ORFV (VEGF-V) and introduced the TRAP gene of S. aureus into the terminus of the ORFV genome to promote TRAP expression in infected keratinocytes. The construction and experimental verification of recombinant ORFV (ORFV-V/TRAP) will provide a reference for in-depth studies on the prevention and control of orf and S. aureus disease.
\end{abstract}

\section{Hosted file}

Construction of recombinant ORFV-TRAP using CRISPR-Cas9 technology.docx available at https : //authorea. com/users/457204/articles/554035-construction-of-recombinant-orfv-trapusing-crispr-cas9-technology 
A

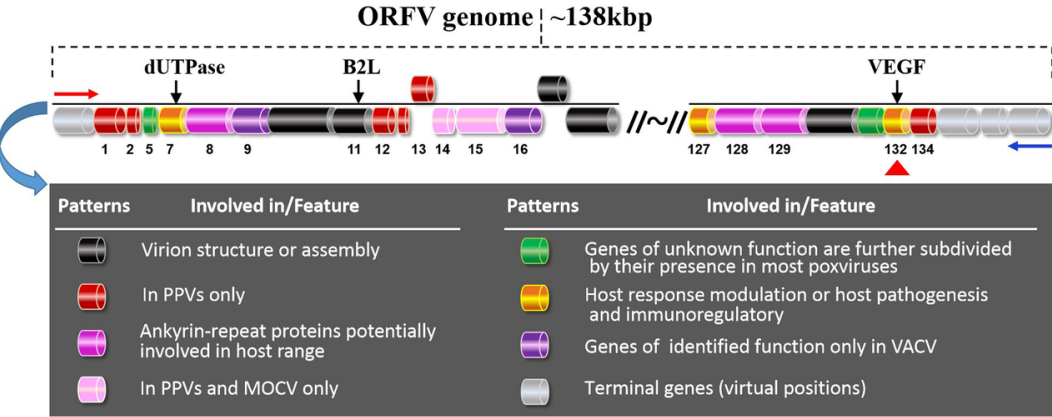

B

C

\begin{tabular}{|c|c|}
\hline SgRNA & Primer sequences \\
\hline Nf1 & $\begin{array}{l}\text { TAATACGACTCACTATAGGG TIT GCCAGAATG } \\
\text { CGTGTCTCGIIITAGAGCTAGA }\end{array}$ \\
\hline Nf2 & $\begin{array}{l}\text { TAATACGACTCACTATAGGGCACACAAAGTGC } \\
\text { GATTGTAT GTTTTAGAGCTAGAA }\end{array}$ \\
\hline Nf3 & $\begin{array}{l}\text { TAATACGACTCACTATAGGGCACCACCACCTA } \\
\text { СGACCACT GITTTAGAGCTAGAA }\end{array}$ \\
\hline Nr1 & $\begin{array}{l}\text { TAATACGACTCACTATAGGGITATCCTGAGAC } \\
\text { ACGCATTCGTTTAGAGCTAGAA }\end{array}$ \\
\hline $\mathrm{Nr2}$ & $\begin{array}{l}\text { TAATACGACTCACTATAGGGACTTGTAACCGA } \\
\text { AACGACTAGITTAGAGCTAGAA }\end{array}$ \\
\hline
\end{tabular}

pX330 modified for gene editing
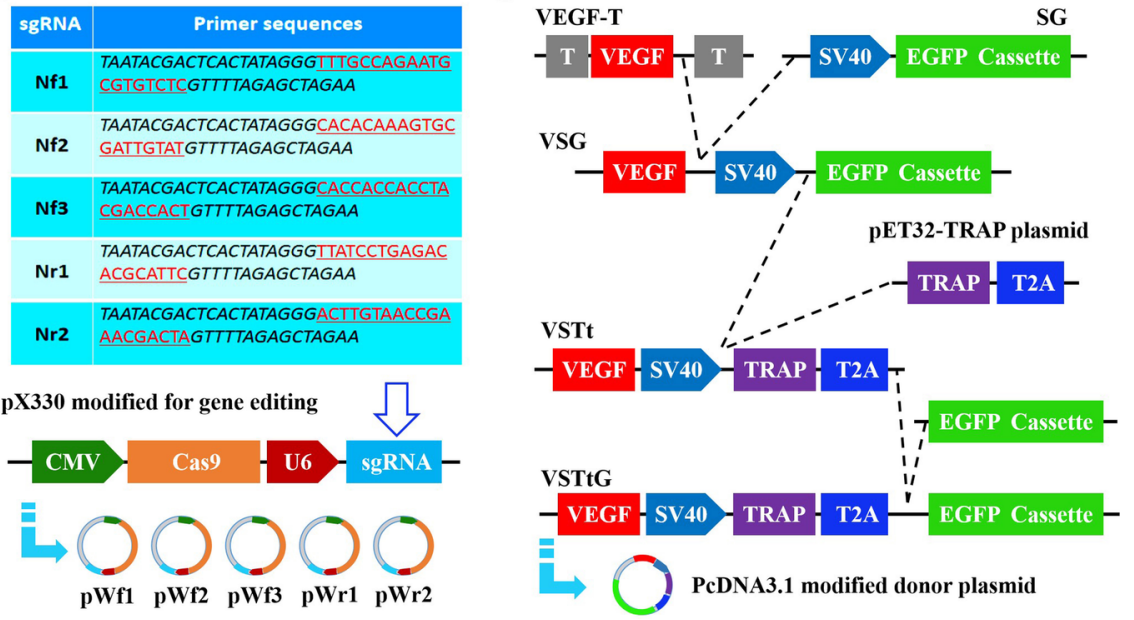

A

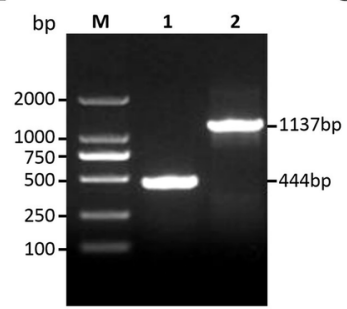

B

Putative amino acid sequence of VEGF

MKLTATIQVVVALLICMYNLPECVSOD NDSPPSTNEWMRTLDKSGCKPRDAV VYLGDEYPENTNLOYNPRCVTVKRCS GCCNGEGQICTAVETRNTTVVVSVTS VSSSSSANGVSSNLQRISLTEHTKCDCI GRTTTPPPTTTREPRR-148aa

\begin{tabular}{|c|c|c|c|c|c|}
\hline \multicolumn{6}{|c|}{ 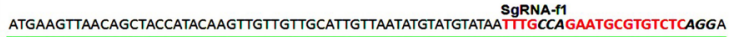 } \\
\hline \multicolumn{6}{|c|}{$\begin{array}{r}\text { TACTTCAATTGTCGATGGTATGTTCAACAACAACGTAACAATTATACATACATATTAAACGG TITIACGCACAGAGTCCTA } \\
\text { SgRNA-r1 GAATGCGTGTCTAGGAT } \\
\text { TAATGATTCACCTCCTTCAACCAATGAATGGATGCGTACACTAGACAAAAGTGGTTGAAACCTAGAGATGCTGTGTT }\end{array}$} \\
\hline \multicolumn{6}{|c|}{ IIACTAAGTGGAGGAAG TTGGTTACTTACCTACGCATGTGATCTGTITCACCAACATTTGGATCTCTACGACAACAAA } \\
\hline \multicolumn{6}{|c|}{$\begin{array}{l}\text { AA } \\
\text { TATTTGGGAGACGAATATCCAGAAAACACTAACCTACAATATAATCCCCGGTGCGTAACTGTTAAACGATGCAGTGGT }\end{array}$} \\
\hline \multicolumn{6}{|c|}{ TAAACCCTCTGCTTATAGGTCTTTTGTGATTGGATGTTATATTAGGGGCCACGCATTGACAATTTGCTACGTCACCAAC } \\
\hline \multicolumn{6}{|c|}{ GCTGTAACGGTGAGGGTCAAATATGCACAGCTGTTGAAACAAGAAATACAACCGTAGTCGTTTCGGTTACAAGTGTGT } \\
\hline \multicolumn{6}{|c|}{$\begin{array}{l}\text { GACATTGCCACTCCCAGTTTATACGTGTCGACAACTTTGTTCTTTATGTTGGCATCAGCAAAGCCAATGTTCACACAG } \\
\text { SgRNA-12 TAGTCGTITCGGTACAAGT } \\
\text { SgRNA-12 } \\
\text { CCAGTTCTTCTAGTGCTAATGGTGTATCTAGTAACCTTCAAAGAATAAGTCTTACAGACACACAAATGCGATGTAT }\end{array}$} \\
\hline \multicolumn{6}{|c|}{ GTCAAGAAGATCACGATTACCACATAGATCATTGGAAGTTTCTTATTCAGAATGTCTTGTGTGTTTCACGCTAACATAAC } \\
\hline \multicolumn{6}{|c|}{$\begin{array}{l}\text { SgRNA-13 } \\
\text { IGGTAGAACAACGACACCACCACTACGACCACTAGGGAACCTAGACGA }\end{array}$} \\
\hline \multicolumn{6}{|c|}{ СATCTTGTTGCTGTGGTGGTGGATGCTGGTGATCCCTTGGATCTGCT } \\
\hline SgRNA name & Nf1 & Nr1 & Nr2 & Nf2 & Nf3 \\
\hline $\begin{array}{l}\text { Position in VEGF } \\
\text { sequence }\end{array}$ & 56 & 63 & 292 & 375 & 409 \\
\hline
\end{tabular}



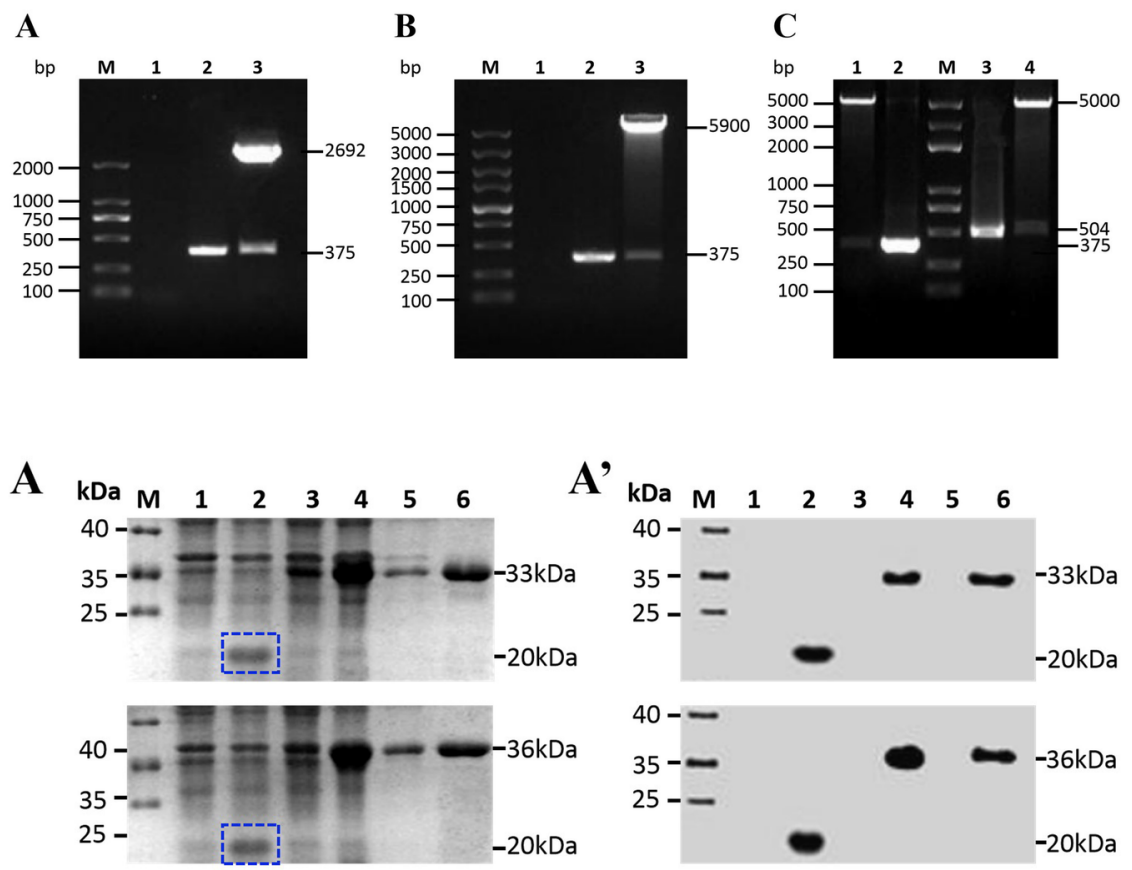

B

B'
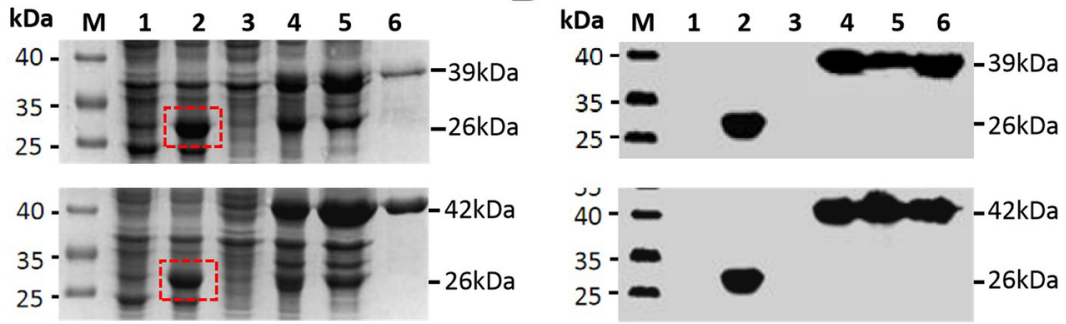

C

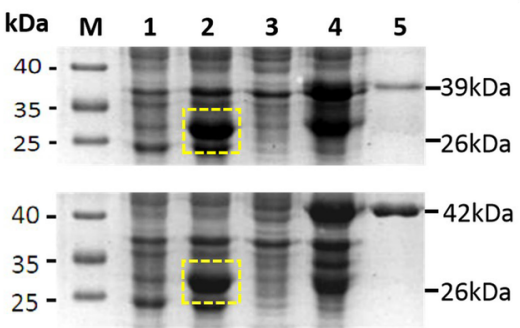

C'

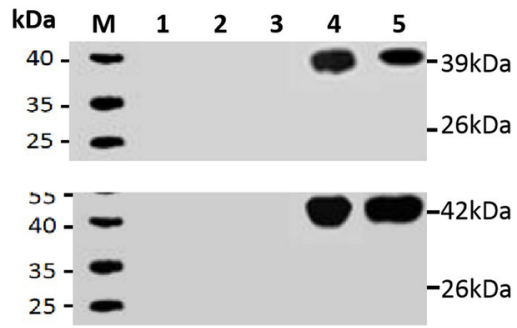



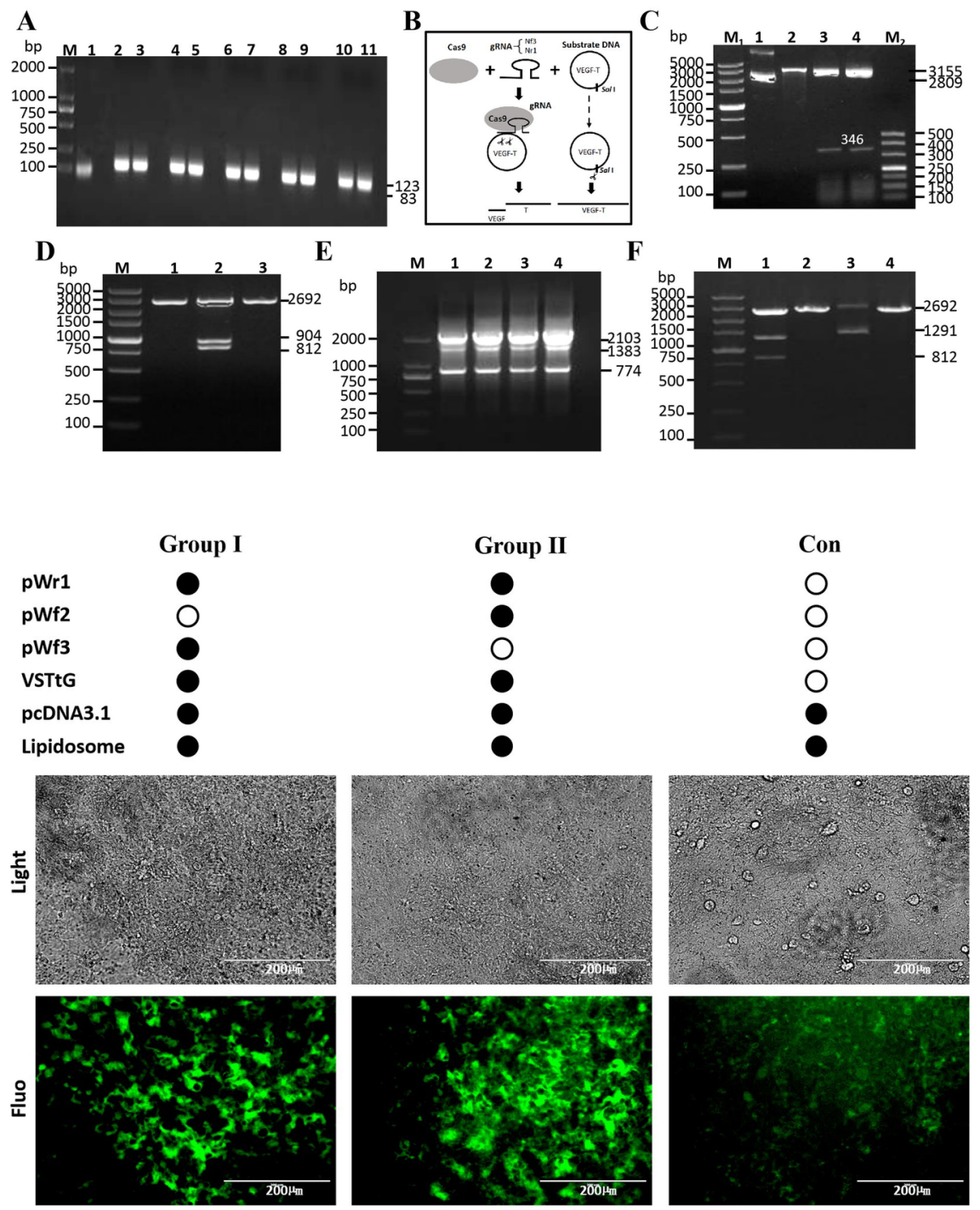


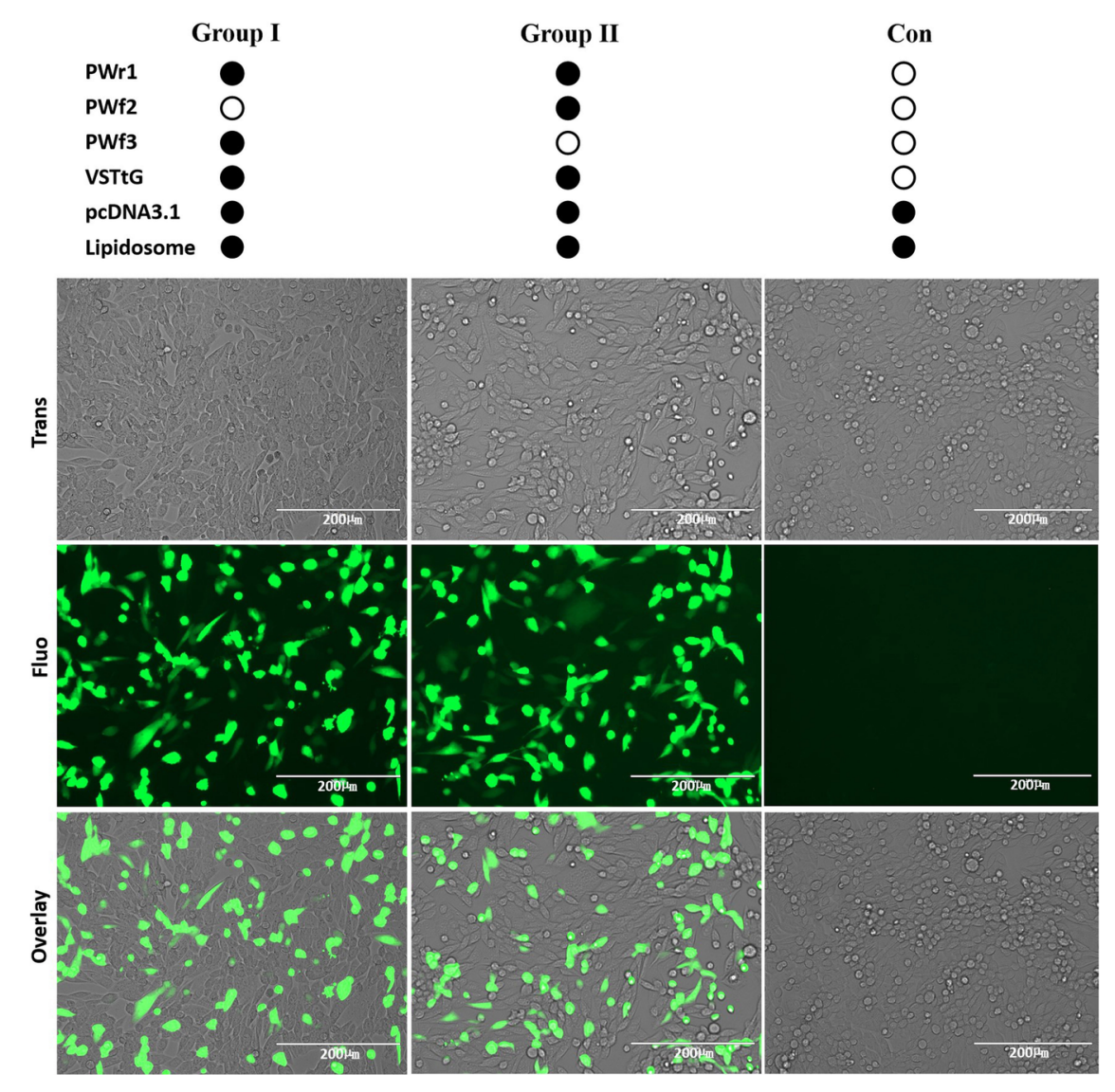




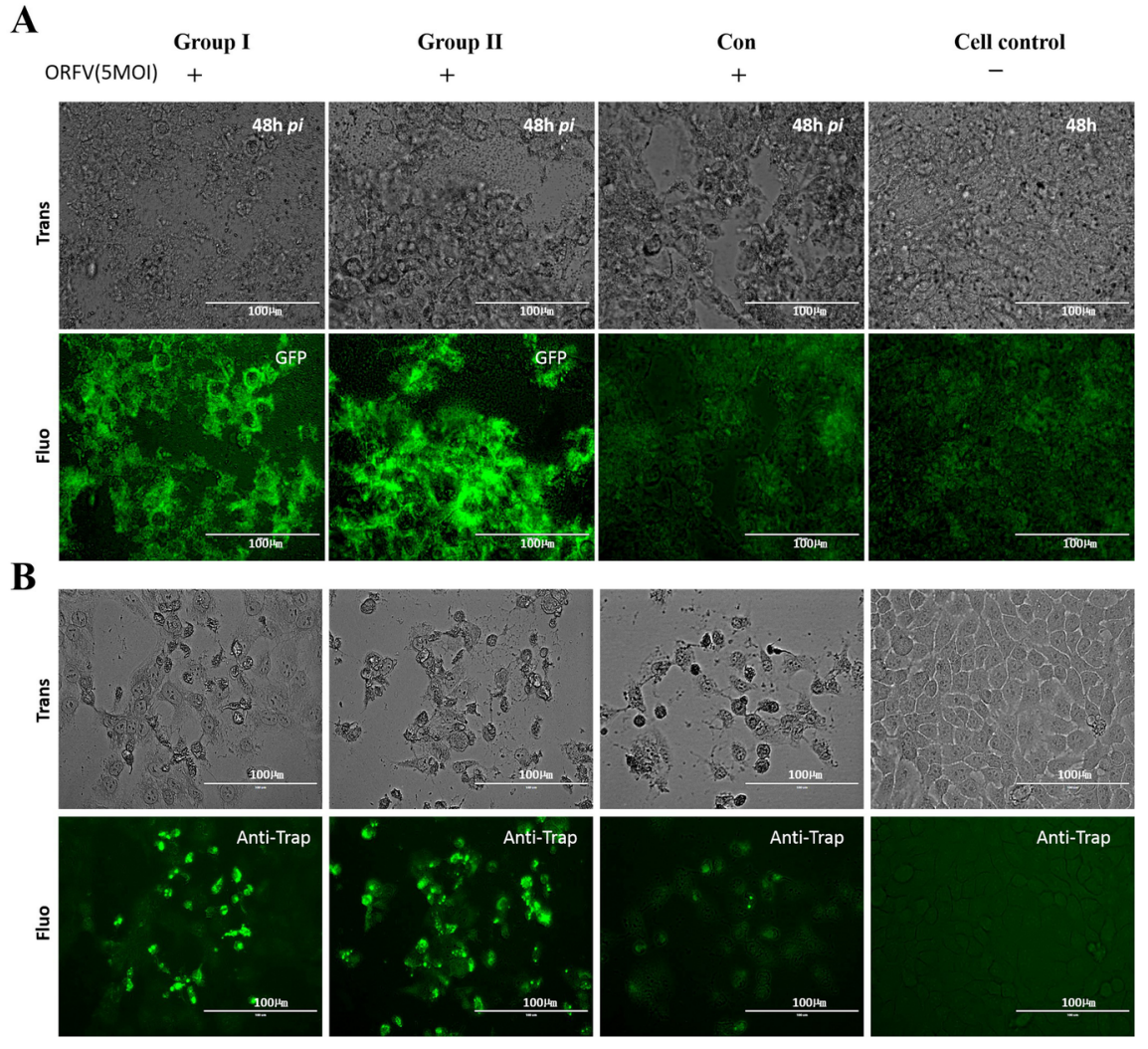

A

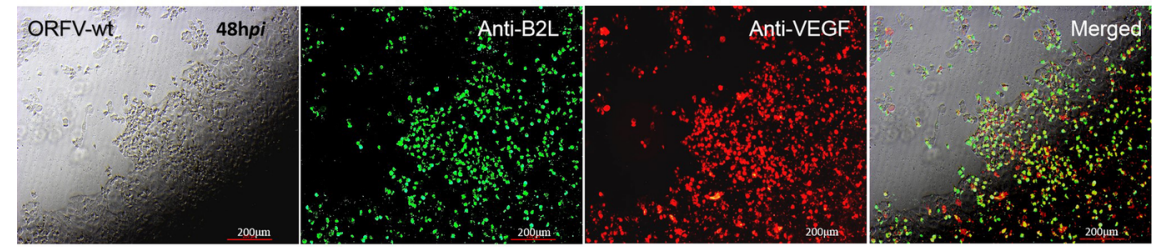

B

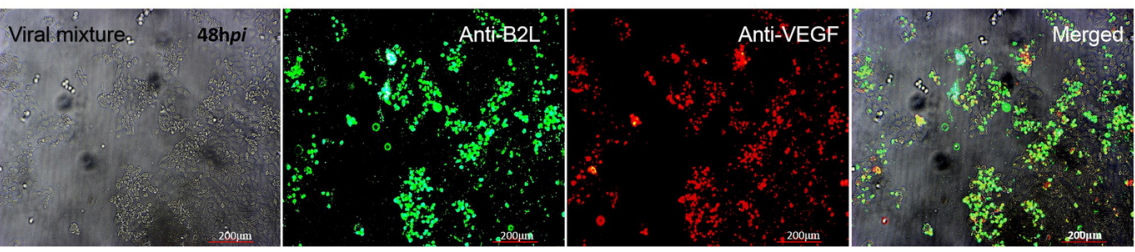


A

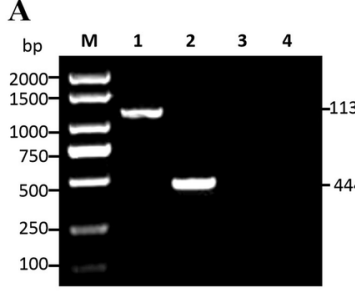

Wild ORFV genome Tamplate - B2L - VEGF .

$\begin{array}{ccccc}\text { Primer } & 1 & 2 & 3 & 4 \\ & & \text { B2L } & \text { VEGF } & \text { TRAP } \\ & & \end{array}$

Band
B

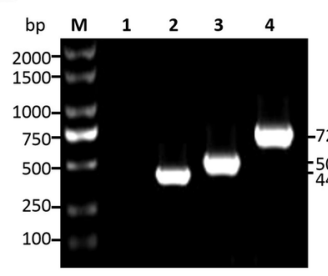

VSTtG-T plasmid - VEGF SV40 TRAP T2A EGFP Cassette-
$\begin{array}{llll}1 & 2 & 3 & 4\end{array}$
B2L VEGF TRAP EGFP
$-+++$

C

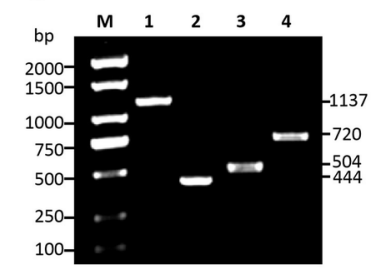

Mixed ORFV genomes - B2L. III VEGE. - VEGF SV40 TRAP T2A EGFP Cassete-

$\begin{array}{llll}1 & 2 & 3 & 4\end{array}$ B2L VEGF TRAP EGFP 